\title{
A NOTE ON THE UNIQUENESS \\ OF THE CLOSED ORBIT \\ OF THE FITZHUGH-NAGUMO SYSTEM
}

\author{
$\mathrm{BY}$ \\ MAKOTO HAYASHI \\ Department of Mathematics, College of Science and Technology, Nihon University, 7-24-1, \\ Narashinodai, Funabashi, 274-8501, Japan
}

\begin{abstract}
A parameter range for which the ordinary differential equations governing the FitzHugh nerve system have a unique nontrivial closed orbit is given. It is wider than those already known.
\end{abstract}

1. Introduction. We consider the following two-dimensional autonomous system of ordinary differential equations:

$$
\left\{\begin{array}{l}
\dot{x}=y-\left\{\frac{1}{3} x^{3}+\eta x^{2}+\left(\eta^{2}-\eta_{0}^{2}\right) x\right\} \\
\dot{y}=-\frac{\rho b}{3}\left\{x^{3}+3 \eta x^{2}+3\left(\eta^{2}+\frac{1}{b}-1\right) x\right\}
\end{array}\right.
$$

where the dot $\left(^{(}\right)$denotes differentiation, $b, \rho$ and $\eta_{0}$ are constants such that $0<b<1$, $0<\rho<1 / b$, and $\eta_{0}=\sqrt{1-\rho b}$, and $\eta$ is some real parameter. The system (1) is called the FitzHugh nerve system and is obtained by transforming the well-known FitzHughNagumo system (see [Fi] and [Na]). It was studied in such papers as [H1], [K-S], [Su], and $[\mathrm{T}-\mathrm{V}]$. We shall prove the following

THEOREM. The system (1) has a unique nontrivial closed orbit if $\eta^{2}<\eta_{0}^{2}$.

This result improves those given in $[\mathrm{H} 1]$ and $[\mathrm{K}-\mathrm{S}]$. In fact, the result that "If either $\eta^{2} \leq 4^{-1} \eta_{0}^{2}$ or $\left\{\rho b^{2}-7 b+6<0\right.$ and $\left.\eta^{2}<\eta_{0}^{2}\right\}$, then the system (1) has a unique nontrivial closed orbit" was given in [K-S]. In [H1] the result that "There is a positive constant $\eta_{1} \leq \eta_{0}$ such that the system (1) has a unique nontrivial closed orbit for $|\eta| \leq \eta_{1}$ " was given. Therefore, the result of the above theorem is clearly stronger than those in [K-S] and [H1] (it was shown in [H1] that the result in [H1] is not included in that of [K-S]). In Sec. 4 it will be shown that there is a concrete case that can be treated by the above theorem, but not by the results in [K-S] and [H1].

Received April 20, 1998.

2000 Mathematics Subject Classification. Primary 34C07, 34C25, 34C26, 34D20.

E-mail address: mhayashi@penta.ge.cst.nihon-u.ac.jp 
2. Lemmas. In this section we prepare some lemmas to be used in the next section to prove the Theorem. We consider the Liénard system of the following form:

$$
\left\{\begin{array}{l}
\dot{x}=y-F(x) \\
\dot{y}=-g(x)
\end{array}\right.
$$

where $F$ is continuously differentiable and $g$ is continuous. We assume the following conditions for the system (2):

[C1] $x g(x)>0$ if $x \neq 0$;

[C2] there exist $a_{1}<0$ and $a_{2}>0$ such that $F\left(a_{1}\right)=F\left(a_{2}\right)=0, x F(x) \leq 0$ for $a_{1}<x a_{2}, F(x)$ is nondecreasing for $x<a_{1}$ and $x>a_{2}$;

[C3] $\lim _{x \rightarrow \pm x} \int_{0}^{x}\left\{F^{\prime}(\xi)+|g(\xi)|\right\} d \xi= \pm \infty$.

To prove the Theorem we shall use the following three lemmas.

Lemma 1. Assume that the system (2) satisfies the conditions [C1], [C2], [C3] and besides

[C4] $G\left(a_{1}\right)>G\left(a_{2}\right)$ and there exists a constant $\alpha \geq 0$ such that $\frac{F(x)}{G^{\curvearrowright}(x)}$ is nondecreasing for $x \in\left(a_{1}, x_{1}\right) \cup\left(a_{2},+\infty\right)$; moreover, there exists a constant $\delta>0$ such that $\frac{F(x)}{G^{\alpha}(x)}$ is strictly increasing in $x$ with $0<|x|<\delta$,

where $G(x)=\int_{0}^{x} g(\xi) d \xi$ and $x_{1}<0$ is a number satisfying the equation $G\left(a_{2}\right)=G\left(x_{1}\right)$. Then the system (2) has a unique nontrivial closed orbit.

Proof of Lemma 1. Under the conditions [C1], [C2], and [C3] the system (2) has at least one nontrivial closed orbit. See [H1] or [H2]. Moreover, by [Ze-1] or [Ze-2], under the conditions [C1], [C2], and [C4] the system (2) has at most one nontrivial closed orbit.

Suppose that the condition $\eta^{2}<\eta_{0}^{2}$ in the Theorem holds with $\eta \geq 0$ (the proof for the case $\eta<0$ is essentially the same).

LEMMA 2. Let

$$
\begin{aligned}
\Gamma(x)=\left\{2 \eta_{0}^{2}+3\left(\frac{1}{b}-1\right)\right\} x^{2}+\eta\left\{2\left(\eta_{0}^{2}-\eta^{2}\right)\right. & \left.+3\left(\eta_{0}^{2}+\frac{1}{b}-1\right)\right\} x \\
& -3\left(\eta^{2}-\eta_{0}^{2}\right)\left(\eta^{2}+\frac{1}{b}-1\right) .
\end{aligned}
$$

Then $\Gamma\left(\varepsilon\left(\eta-\eta_{0}\right)\right)>0$ if $\eta^{2}<\eta_{0}^{2}$, where

$$
\varepsilon=\frac{3\left(\eta+\eta_{0}\right)\left(\eta^{2}+\frac{1}{b}-1\right)}{2 \eta_{0}\left\{2\left(\eta_{0}^{2}-\eta^{2}\right)+3\left(\eta_{0}^{2}+\frac{1}{b}-1\right)\right\}}
$$


Proof of Lemma 2. If $\eta^{2}<\eta_{0}^{2}$, we have

$$
\begin{aligned}
\Gamma\left(\varepsilon\left(\eta-\eta_{0}\right)\right)= & \varepsilon^{2}\left(\eta-\eta_{0}\right)^{2}\left\{2 \eta_{0}^{2}+3\left(\frac{1}{b}-1\right)\right\} \\
& +\varepsilon \eta\left\{2\left(\eta_{0}^{2}-\eta^{2}\right)+3\left(\eta_{0}^{2}+\frac{1}{b}-1\right)\right\}\left(\eta-\eta_{0}\right)-3\left(\eta^{2}-\eta_{0}^{2}\right)\left(\eta^{2}+\frac{1}{b}-1\right) \\
= & \varepsilon^{2}\left(\eta-\eta_{0}\right)^{2}\left\{2 \eta_{0}^{2}+3\left(\frac{1}{b}-1\right)\right\} \\
& +3\left(\frac{\eta}{2 \eta_{0}}-1\right)\left(\eta^{2}-\eta_{0}^{2}\right)\left(\eta^{2}+\frac{1}{b}-1\right)>0 .
\end{aligned}
$$

LEMMA 3. Let

$$
g(x)=\frac{\rho b}{3}\left\{x^{3}+3 \eta x^{2}+3\left(\eta^{2}+\frac{1}{b}-1\right) x\right\} \quad \text { and } \quad G(x)=\int_{0}^{x} g(\xi) d \xi
$$

Then $G(a)-G(-a) \geq 0$ for every $a>0$.

Proof of Lemma 3. Since $G(a)-G(-a)=\frac{2}{3} \rho b \eta a^{3} \geq 0$, the proof is completed.

3. Proof of Theorem. We shall prove the Theorem by using the above three lemmas. We set $F(x)=(1 / 3) x^{3}+\eta x^{2}+\left(\eta^{2}-\eta_{0}^{2}\right) x$. Then, if $\eta^{2}<\eta_{0}^{2}$, we see easily that the system (2) satisfies the conditions [C1], [C2], and [C3]. We shall check the condition [C4] in Lemma 1. We have for $\eta^{2}<\eta_{0}^{2}$,

$$
a_{1}=\frac{-3 \eta-\sqrt{12 \eta_{0}^{2}-3 \eta^{2}}}{2}<0 \quad \text { and } \quad a_{2}=\frac{-3 \eta+\sqrt{12 \eta_{0}^{2}-3 \eta^{2}}}{2}>0
$$

Then we get

$$
G\left(a_{1}\right)-G\left(a_{2}\right)=\frac{\rho b}{4}\left\{\eta^{3}+2 \eta_{0}^{2}+6\left(\frac{1}{b}-1\right)\right\} \sqrt{12 \eta_{0}^{2}-3 \eta^{2}}>0
$$

If $\eta^{2}<\eta_{0}^{2}$, since $0<\varepsilon<1$, we have $a_{2}>\frac{3}{2}\left(\eta_{0}-\eta\right)>\varepsilon\left(\eta_{0}-\eta\right)$. Let $x_{1}<0$ be a number satisfying the equation $G\left(a_{2}\right)=G\left(x_{1}\right)$. From the above fact, the monotonicity of $G$ and Lemma 3, it follows that

$$
G\left(x_{1}\right)=G\left(a_{2}\right)>G\left(\varepsilon\left(\eta_{0}-\eta\right)\right) \geq G\left(\varepsilon\left(\eta-\eta_{0}\right)\right) .
$$

Using the fact that $a_{1}<x_{1}<\varepsilon\left(\eta-\eta_{0}\right)<0$, we shall show that $F(x) / G^{\alpha}(x)$ is nondecreasing for $x \in\left(a_{1}, \varepsilon\left(\eta-\eta_{0}\right)\right) \cup\left(a_{2},+\infty\right)$. This means that $F^{\prime}(x) G(x)-\alpha F(x) g(x) \geq 0$ for $x \in\left(a_{1}, \varepsilon\left(\eta-\eta_{0}\right)\right) \cup\left(a_{2},+\infty\right)$. 
From the calculation in $[\mathrm{H} 1]$ (see $[\mathrm{H} 1]$, p. 333 ), we see that the above claim means that

$$
\begin{aligned}
\Phi(x, \alpha)= & (3-4 \alpha) x^{4}+6 \eta(3-4 \alpha) x^{3} \\
& +3\left\{5(3-4 \alpha) \eta^{2}-(1-4 \alpha) \eta_{0}^{2}+2(3-2 \alpha)\left(\frac{1}{b}-1\right)\right\} x^{2} \\
& +12 \eta\left\{2(2-3 \alpha) \eta^{2}-(1-3 \alpha) \eta_{0}^{2}+3(1-\alpha)\left(\frac{1}{b}-1\right)\right\} x \\
& +18\left(\eta^{2}-\eta_{0}^{2}\right)\left(\eta^{2}+\frac{1}{b}-1\right)(1-2 \alpha) \\
\geq & 0
\end{aligned}
$$

for $x \in\left(a_{1}, \varepsilon\left(\eta-\eta_{0}\right)\right) \cup\left(a_{2},+\infty\right)$.

Let $\alpha=\frac{3}{4}$. Thus we get the following expression, which is of degree 2 in $x$ :

$$
\begin{aligned}
& \Phi\left(x, \frac{3}{4}\right) \\
& =3\left[\left\{2 \eta_{0}^{2}+3\left(\frac{1}{b}-1\right)\right\} x^{2}+\eta\left\{2\left(\eta_{0}^{2}-\eta^{2}\right)+3\left(\eta_{0}^{2}+\frac{1}{b}-1\right)\right\} x\right. \\
& \left.-3\left(\eta^{2}-\eta_{0}^{2}\right)\left(\eta^{2}+\frac{1}{b}-1\right)\right] \\
& =3 \Gamma(x) .
\end{aligned}
$$

If $\eta^{2}<\eta_{0}^{2}$, from the fact that $\Gamma$ is a function of degree 2 , the inequality $\Gamma(0)>0$ and Lemma 2, we conclude that $\Phi\left(x, \frac{3}{4}\right) \geq 0$ for $x \in\left(a_{1}, \varepsilon\left(\eta-\eta_{0}\right)\right) \cup\left(a_{2},+\infty\right)$. Therefore, the condition [C4] in Lemma 1 is satisfied.

4. A numerical example. We shall present the phase portrait of the following system as an example illustrating the application of the Theorem. We consider the system (1) with $b=1 / 2, \rho=1$, and $\eta^{2}=3 / 8$ :

$$
\left\{\begin{array}{l}
\dot{x}=y-\left(\frac{1}{3} x^{3}+\frac{\sqrt{6}}{4} x^{2}-\frac{1}{8} x\right), \\
\dot{y}=-\frac{1}{6}\left(x^{3}+\frac{3 \sqrt{6}}{4} x^{2}+\frac{33}{8} x\right) .
\end{array}\right.
$$

In this case, since $\eta_{0}^{2}=1-\rho b=1 / 2>\eta^{2}$, the system (3) satisfies the condition in the Theorem. Thus we see that the system (3) has a unique nontrivial closed orbit; see Figure 1. We note that this system does not satisfy the condition in [H1] nor that of [K-S], either.

REMARK. In [Su] it was shown that the system (1) has no nontrivial closed orbits if it satisfies the conditions

$$
\eta^{2} \geq \eta_{0}^{2} \text { and } \eta^{4}-4 \eta^{2} \eta_{0}^{2}+\eta_{0}^{4}+2\left(\frac{1}{2}-1\right) \eta^{2}-4\left(\frac{1}{b}-1\right) \eta_{0}^{2}+4\left(\frac{1}{b}-1\right)^{2} \geq 0
$$

or

$$
2\left\{\eta_{0}^{2}+\left(\frac{1}{b}-1\right)\right\}^{3}<\eta^{2}\left\{\eta^{2}+3\left(\frac{1}{b}-1\right)\right\}^{2}
$$


After that, Treskov and Volokitin improved the result of $[\mathrm{Su}]$ by applying bifurcation theory. It is difficult to give the condition by the explicit formulas (see [T-V]).

We do not know yet what happens in the region in the $\left(\eta, \eta_{0}\right)$-plane in which $\eta^{2} \geq \eta_{0}^{2}$, but the condition of $[\mathrm{Su}]$ or $[\mathrm{T}-\mathrm{V}]$ is not satisfied. But some numerical experiments tell us that the system may have two (or more) nontrivial closed orbits if $\left(\eta, \eta_{0}\right)$ is in the above-mentioned region. For instance, consider the system (1) with $b=\frac{5}{6}, \rho=\frac{93}{250}$, and $\eta^{2}=\frac{7}{10}$. It satisfies neither the condition $[\mathrm{Su}]$ nor $[\mathrm{T}-\mathrm{V}]$. From the phase portrait of the system it seems that it has several nontrivial closed orbits. We hope to show this mathematically in the future.

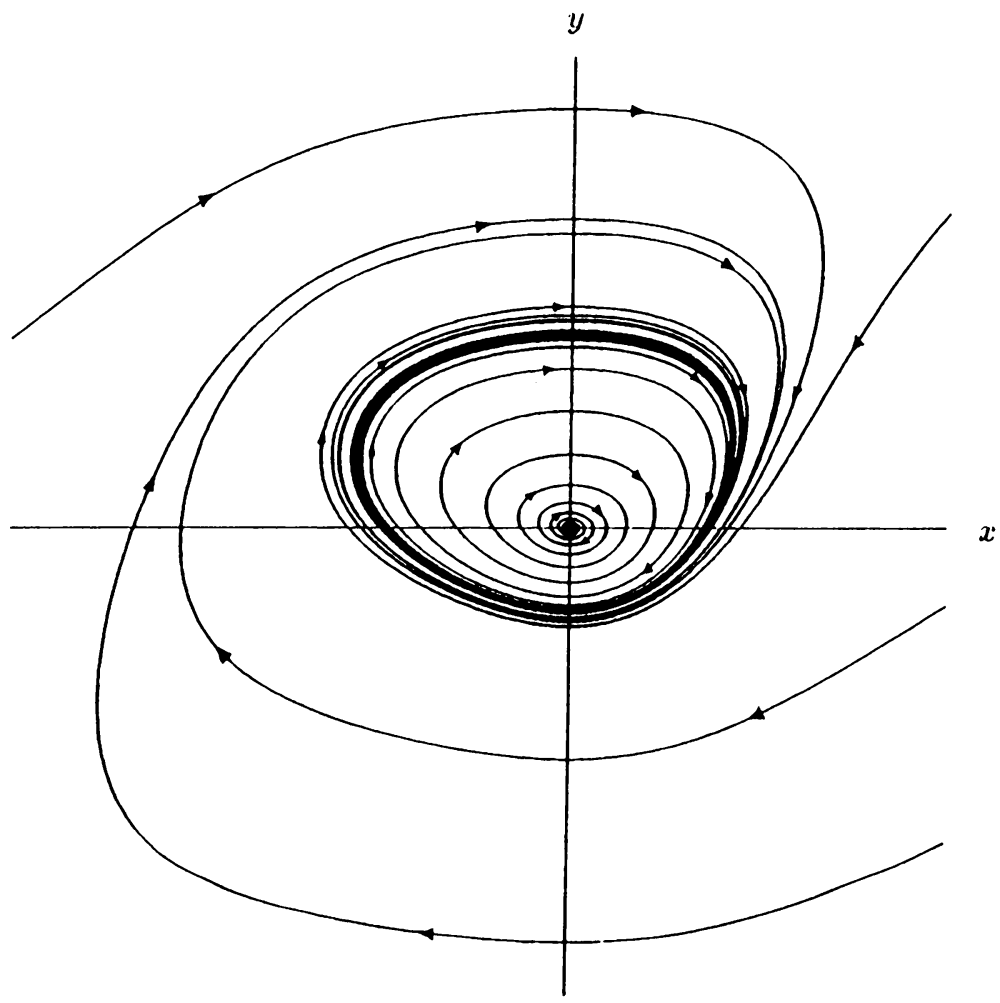

FIG. 1

Acknowledgment. The author would like to express his sincere gratitude to Professor Takesi Yamanaka at Nihon University for having given him continuous encouragement.

\section{REFERENCES}

[Fi] R. FitzHugh, Impulses and physiological states in theoretical models of nerve membrane, Biophys. J. 1, 445-466 (1961)

[H1] M. Hayashi, On the uniqueness of the closed orbit of FitzHugh-Nagumo system, Math. Japon. 46 (2), 331-336 (1997) 
[H2] M. Hayashi, On the uniqueness of the closed orbit of the Liénard system, Math. Japon. 46 (3), 371-376 (1997)

[K-S] E. Kaumann and U. Staude, Uniqueness and nonexistence of limit cycles for the FitzHugh equation, Lecture Notes in Math. 1017, Springer-Verlag, New York, 1983, pp. 313-321

[Na] J. Nagumo, S. Arimoto, and S. Yoshizawa, An active pulse transmission line simulating nerve axon, Proc. IRE 50, 2061-2070 (1962)

[Su] J. Sugie, Nonexistence of periodic solutions for the FitzHugh nerve system, Quart. Appl. Math. 49, 543-554 (1991)

[T-V] S. A. Treskov and E. P. Volokitin, On existence of periodic orbits for the FitzHugh nerve system, Quart. Appl. Math. 54, 601-607 (1996)

[Ze-1] X. Zeng, On the uniqueness of limit cycle of Liénard's equation, Scientia Sinica (Series A) 25, 583-592 (1982)

[Ze-2] X. Zeng, Z. Zhang, and S. Gao, On the uniqueness of the limit cycle of the generalized Liénard equation, Bull. London Math. Soc. 26, 213-247 (1994) 